\title{
"RIESGO AUMENTADO DEL DESARROLLO DE UN SEGUNDO MELANOMA CUTÁNEO PRIMARIO SOBRE UN NEVO EN PACIENTES DIAGNOSTICADOS PREVIAMENTE DE MELANOMA CUTÁNEO PRIMARIO SOBRE NEVO."
}

$$
\text { Por }
$$

\section{Begoña Echeverría García}

Médico Interno Residente de Dermatología

Fundación Instituto Valenciano de Oncología

\section{Carlos Ferrándiz Foraster.}

Catedrático de Dermatología. Universidad Autónoma de Barcelona.

Jefe de Servicio de Dermatología. Hospital Universitario Germans Trias i Pujol

Eduardo Nagore Enguíanos.

Jefe Clínico de Dermatología.

Fundación Instituto Valenciano de Oncología.

TREBALL DE RECERCA

SETEMBRE 2010 


\section{$\underline{\text { ÍNDICE }}$}

$\begin{array}{ll}\text { 1. RESUMEN } & \text { Pág. } 3\end{array}$

1.1.- Antecedentes

1.2.- Objetivos

1.3.- Métodos

1.4.- Resultados

1.5.- Conclusiones

$\begin{array}{ll}\text { 2. INTRODUCCIÓN } & \text { Pág. } 4\end{array}$

3. PACIENTES Y MÉTODOS Pág. 5

$\begin{array}{ll}\text { 4. RESULTADOS } & \text { Pág. } 7\end{array}$

$\begin{array}{ll}\text { 5. DISCUSIÓN } & \text { Pág. } 10\end{array}$

$\begin{array}{ll}\text { 6. CONCLUSIONES } & \text { Pág. } 13\end{array}$

$\begin{array}{ll}\text { 7. TABLAS Y GRÁFICOS } & \text { Pág. } 14\end{array}$

$\begin{array}{ll}\text { 8. BIBLIOGRAFÍA } & \text { Pág. } 20\end{array}$ 


\section{RESUMEN}

Antecedentes: Los pacientes diagnosticados de un melanoma cutáneo tienen más riesgo que el resto de la población de padecer nuevos melanomas a lo largo de su vida. La mayoría de estos melanomas aparecen "de novo", pero existe un porcentaje de ellos que se desarrolla sobre nevos melanocíticos previos.

Objetivo: Determinar los factores de riesgo asociados al desarrollo de un segundo melanoma cutáneo sobre nevo en pacientes diagnosticados de melanoma cutáneo.

Métodos: Se seleccionaron los datos de 981 pacientes diagnosticados de un primer melanoma cutáneo, 47 de los cuales habían sido diagnosticados de un segundo melanoma. Todos los pacientes fueron clasificados en función de si el melanoma presentó o no asociación histológica a un nevo.

Resultados: La edad inferior a 40 años, la localización en el tronco y el subtipo histológico de extensión superficial, fueron los factores de riesgo independientes asociados al desarrollo de un primer melanoma sobre nevo. El único factor asociado al desarrollo de un segundo melanoma sobre nevo fue que el primer melanoma también se hubiera presentado asociado a un nevo (OR: 9,51, 95\% de intervalo de confianza: 1.6$56,56 ; \mathrm{p}=0,042)$.

Conclusiones: Los melanomas se desarrollan asociados a un nevo en pacientes jóvenes, en el tronco y del subtipo de extensión superficial. A su vez, el desarrollo de un primer melanoma sobre nevo aumenta el riesgo hasta 9 veces de desarrollar un segundo melanoma sobre un nevo.

Estos hallazgos, reiteran la importancia del seguimiento estrecho de las lesiones melánicas en pacientes que han desarrollado un primer melanoma sobre nevo. 


\section{INTRODUCCIÓN}

Desde un punto de vista clínico y epidemiológico, se ha propuesto que existen al menos dos vías etiopatogénicas diferentes para explicar el desarrollo de un melanoma. En el año 1998, Whiteman y cols. sugirieron que, por una parte, existía una vía que traducía una inestabilidad de las células pigmentarias y, por otra parte, otra vía que sería el resultado de una exposición solar crónica ${ }^{1}$. La primera vía se caracteriza por la presencia de múltiples nevos y efélides y la segunda por el desarrollo de cáncer cutáneo no melanoma y la expresión de la proteína p53 en el tumor primario.

Más recientemente, se ha constatado que los melanomas en zonas de exposición solar intermitente presentan frecuentemente mutaciones en BRAF y en NRAS y los melanomas localizados en zonas no fotoexpuestas, por ejemplo los melanomas mucosos y acrales, muestran mutaciones en C-KIT y en CCDN1 ${ }^{2}$.

La existencia de una vía de inestabilidad de las células pigmentarias, en la que se asume que hay una susceptibilidad individual a la proliferación melanocítica, también se apoya en el riesgo de desarrollar melanomas en los pacientes con múltiples nevos, tanto comunes como atípicos. Por otra parte, aproximadamente hasta un $20 \%$ de los melanomas cutáneos se encuentran asociados a un nevo ${ }^{3,4}$. En algunos estudios, se ha observado que estos melanomas asociados a nevos aparecen con más frecuencia en el tronco de pacientes más jóvenes y con una mayor densidad de nevos que los melanomas no asociados a nevos y que suelen ser del subtipo de extensión superficial ${ }^{3,4}$. Estos hechos sugieren que los pacientes que desarrollan un melanoma sobre un nevo lo harían mediante la vía de la inestabilidad de las células pigmentarias.

Puesto que se sabe que los pacientes que han desarrollado un primer melanoma cutáneo tienen más riesgo de desarrollar un segundo melanoma cutáneo y que muchos de éstos presentan múltiples nevos, en este estudio se plantea la hipótesis de que los pacientes que desarrollan un segundo melanoma es más probable que lo hagan sobre un nevo si pertenecen a la vía de la inestabilidad de células pigmentarias ${ }^{5-8}$.

El objetivo principal de este estudio es conocer los factores de riesgo de presentar un segundo melanoma cutáneo sobre un nevo. De forma secundaria se busca identificar los factores de riesgo asociados al desarrollo de un primer melanoma sobre un nevo en nuestra población. 


\section{PACIENTES Y MÉTODOS}

Se diseñó un estudio observacional retrospectivo a partir de los datos incluidos en la base de datos de melanoma del Servicio de Dermatología del Instituto Valenciano de Oncología. Las características de dicha base de datos ya han sito descritas con anterioridad $^{9}$.

Para el estudio se incluyeron los pacientes diagnosticados de melanoma cutáneo entre el 1 de enero de 1990 y el 31 de mayo de 2009. Se excluyeron aquellos pacientes con melanoma metastásico de tumor primario desconocido y aquellos cuyo primer melanoma fue diagnosticado previamente a la fecha definida anteriormente.

Se obtuvo un total de 981 pacientes, de los cuales 47 presentaron más de un melanoma cutáneo.

Para la identificación de los factores de riesgo asociados al desarrollo de un melanoma sobre un nevo se seleccionaron los 981 pacientes diagnosticados de melanoma cutáneo. Para el estudio de los factores asociados al desarrollo de un segundo melanoma sobre un nevo se seleccionaron los 47 pacientes diagnosticados de más de un melanoma cutáneo. Los pacientes afectos de un solo melanoma fueron clasificados en función de si éste presentaba o no un nevo en el estudio histológico. Se definió como melanoma sobre nevo (MSN) al melanoma en el que se observaba en el estudio histológico la presencia de nidos, cordones o hileras aisladas de células névicas citológicamente benignas en la dermis adyacente o por debajo del melanoma ${ }^{10}$. En caso contrario, se consideró que el melanoma había aparecido "de novo" (MDN) sobre piel sin lesión melanocítica asociada. Los pacientes diagnosticados de más de un melanoma fueron clasificados de igual manera en función de si el segundo melanoma se presentó en asociación a un nevo o no.

Para el análisis comparativo se consideraron las siguientes variables:

1. Epidemiológicas: Sexo, edad $(<40,40-60,>60)$ y localización del melanoma (cabeza/cuello, miembros superiores o inferiores y acrales).

2. Fenotípicas: Fototipo (I-II/III-IV), color de ojos (oscuros/claros) y del pelo (moreno/ castaño, rubio o pelirrojo) y número de nevos comunes $(<20,20-50,51-100$ y $>100)$ o atípicos (alguno/ninguno).

3. Factores ambientales: Años de exposición solar laboral $(0,<20,>20)$ y antecedentes de quemaduras solares severas tanto en general $(0,1-5,6-10,>10)$ como en la zona del melanoma (no, leves, severas). 
4. Antecedentes familiares de melanoma en primer o segundo grado (sí, no).

5. Histológicas: Presencia de restos de nevo, índice de Breslow ( $<$ ó igual a $1.00 \mathrm{~mm}$, $1.01-4.00 \mathrm{~mm},>4.00 \mathrm{~mm}$ ) y subtipo histológico del primer melanoma (extensión superficial, nodular, sobre léntigo maligno y lentiginoso acral).

La forma de recogida de los datos y la codificación de los mismos ha sido descrita con detalle anteriormente ${ }^{9}$.

Las diferencias entre las distribuciones de cada variable en cada una de las categorías fueron evaluadas mediante la prueba de la Ji cuadrado de Pearson. Se calcularon los valores de las odds ratio (OR) mediante regresión logística tanto univariada como multivariada. La importancia relativa de los factores pronósticos se midió mediante el valor de Ji cuadrado, basada en la prueba de Wald del coeficiente asociado con cada factor pronóstico en el modelo de regresión. Los factores que obtuvieron mayores valores para la Ji cuadrado fueron más significativos en cada modelo. Se usó el valor de la Ji cuadrado para ordenar los factores pronóstico, ya que su interpretación no se relaciona con la codificación de la covariable. Los valores de probabilidad usados para la identificación de la heterogeneidad entre grupos se ajustaron para comparaciones múltiples usando la corrección de Bonferroni. El estudio estadístico se realizó con el paquete estadístico incluido en el programa SPSS, versión 15.0. 


\section{RESULTADOS}

De los 981 pacientes seleccionados para el estudio, 456 eran hombres (46,5\%) y 525 eran mujeres (53,5\%) (Gráfico 1), con una edad mediana en el diagnóstico de 54 años (intervalo intercuartílico de 40 y 67 años) y una mediana de seguimiento de 46 meses (intervalo intercuartílico de 19 y 90 meses). Del grupo de los pacientes seleccionados, 297 (30,3\%) desarrollaron un MSN y 684 (69,7\%) presentaron un MDN (Gráfico 2).

Durante el seguimiento, 47 pacientes desarrollaron un segundo melanoma. En 11 de los pacientes $(24,4 \%)$ este segundo melanoma se desarrolló sobre un nevo y en $34(75,6 \%)$ apareció de novo (Gráfica 3). En dos de los casos, en los que el segundo melanoma fue extirpado en otro centro, no se pudo recuperar la información acerca de la presencia o no de restos de nevo junto al melanoma.

El análisis de los factores relacionados con la presencia de un nevo asociado a melanoma en el grupo general de 981 pacientes diagnosticados de un melanoma (Tabla 1) mostró que la edad no estaba distribuida de forma homogénea entre los dos grupos, MSN y MDN ( $\mathrm{p}=0,0016)$. Se constató un predominio de pacientes jóvenes en el grupo con MSN. Así, por ejemplo, el 35,4\% de los pacientes que desarrollaron un MSN lo hizo a una edad inferior a los 40 años frente al 19,4\% de los pacientes que desarrollaron un MDN en el mismo grupo de edad. De igual manera, los pacientes de más de 60 años tenían 66\% menos de probabilidad de presentar un MSN respecto a los pacientes con menos de 40 años (OR 0,34, IC 95\% OR 0,24-0,49).

La presencia de algún nevo clínicamente atípico también mostró una asociación estadísticamente significativa con el desarrollo de un $\operatorname{MSN}(\mathrm{p}=0,0016)$. Mientras que un $30,2 \%$ de los pacientes con MSN presentaba algún nevo atípico, sólo un 18,2\% de los pacientes con MDN tenían esta característica. Los pacientes que presentaban algún nevo atípico tenían casi el doble de riesgo de desarrollar un MSN respecto a los pacientes que carecían de algún nevo atípico. (OR 1,94; IC 95\% OR 1,40-2,71).

Los MSN no se distribuían de forma homogénea según la localización ( $\mathrm{p}=0,0016)$. Más de la mitad de los MSN se localizaban en el tronco (54,9\%) frente a sólo el 33,8\% de los MDN que presentaban esta localización.

Finalmente, el análisis de los subtipos histológicos de melanoma mostró una predilección del subtipo histológico de extensión superficial en los pacientes que desarrollaron un MSN ( $p=0,0016)$. El porcentaje de pacientes con un MSN que desarrollaron este subtipo histológico fue de un 79,1\% frente al 60,9\% de los pacientes 
con MDN. El riesgo de que un paciente con MSN desarrollase un melanoma del subtipo de extensión superficial fue del 3,55 respecto a los del tipo léntigo maligno melanoma (OR 3,55, IC 95\% de OR 1,57-8,01).

No se observaron diferencias significativas entre ambos grupos respecto al fototipo, el color de los ojos, el color del pelo, el número de nevos comunes, el sexo, el número de quemaduras solares severas (tanto generales como sobre el área del melanoma), los años de exposición solar crónica, el espesor tumoral ni los antecedentes personales de melanoma (Tabla 1). Para la valoración del número de nevos como factor de riesgo de desarrollar un MSN se evaluó, además, su valor al categorizar post-hoc la variable en > ó igual a 20 nevos respecto a $<20$, y en $>50$ nevos respecto a $>$ ó igual a 50 . En ambos casos, tras la corrección de Bonferroni, no hubo significación estadística aunque la relación fue mayor cuando se estableció el punto de corte en los 20 nevos comunes.

Tras el estudio multivariado ajustado por sexo, sólo la edad, la localización y el tipo histológico se mantuvieron en el modelo como las variables predictivas independientes relacionadas con el desarrollo de un MSN (Tabla 2). Por orden de importancia, de acuerdo con el valor de la Ji cuadrado (prueba de Wald), se observó que la localización en el tronco, la edad menor de 40 años y el tipo histológico de extensión superficial, son las características más relacionadas con la presentación de un MSN. (Gráfica 4)

Para el estudio de los factores relacionados con el desarrollo de un segundo MSN, se seleccionaron sólo aquellas variables que fueron significativas en el estudio univariado del desarrollo de un MSN más la variable presencia o ausencia de nevo en el primer melanoma.

El análisis de los 47 pacientes diagnosticados de más de un melanoma, mostró que de entre todas las variables a estudio, sólo haber presentado un primer melanoma en asociación histológica a un nevo se relacionaba con el desarrollo de un segundo MSN $(p=0,042)$ (Tabla 3). El 62,5\% de los pacientes que presentaron un segundo MSN también habían mostrado esta asociación en el primer melanoma, mientras que sólo el $16,2 \%$ de los pacientes que habían desarrollado un primer MDN desarrollaba un segundo MSN. El riesgo del desarrollo de un segundo MSN cuando se había presentado previamente un primer MSN fue de 9,51 frente a los pacientes que presentaron el primer MDN. (OR 9,51, IC 95\% de OR: 1,60-56,56)

A diferencia del grupo general de 981 pacientes, no se observó una mayor relación del desarrollo de un segundo MSN con la edad de presentación más joven que en el MDN, 
la presencia de nevos clínicamente atípicos, la localización en el tronco y el subtipo histológico de extensión superficial. 


\section{DISCUSIÓN}

Teniendo en cuenta que los pacientes que han padecido un primer melanoma cutáneo tienen más riesgo que la población general de desarrollar un segundo melanoma cutáneo $^{5,7,11}$, y que muchos de estos pacientes forman parte del grupo descrito por Whiteman y col. ${ }^{1}$ caracterizado por la presencia de múltiples nevos (inestabilidad de células melánicas), este estudio fue realizado con el fin de buscar unas características clínicas, epidemiológicas e histológicas que ayuden a identificar a pacientes que hayan sido previamente diagnosticados de un primer melanoma cutáneo y que tengan más riesgo de desarrollar un segundo melanoma sobre un nevo pre-existente. Estos pacientes serían los que más se beneficiarían de un seguimiento estrecho de sus lesiones melánicas benignas.

En la valoración de los resultados de este estudio hay que tener en cuenta una serie de limitaciones. Para el estudio de los factores de riesgo asociados al desarrollo de un MSN, se seleccionaron y analizaron los datos de pacientes diagnosticados de un melanoma cutáneo desde el año 1990 hasta el 2009. Los datos del periodo de tiempo comprendido entre el año 1990 y el 1999 fueron recogidos de forma retrospectiva pero a partir del año 2000 de forma prospectiva. Para evaluar si el sesgo de supervivencia afectaba a los resultados del estudio y aumentar el valor interno del mismo, se compararon todos los datos evaluados entre los pacientes del periodo 1990-1999 y los pacientes diagnosticados entre 2000 y 2009 y no se encontraron diferencias significativas (datos no mostrados en este estudio). Por otra parte, cabe señalar que todos los datos fueron recogidos en todos los casos con criterios homogéneos y por un mismo observador. Se asume el sesgo de memoria que pueden tener los pacientes para recordar el número de quemaduras tanto globales como en la zona del melanoma así como la intensidad de las mismas. Del mismo modo, hay que asumir el posible sesgo de clasificación introducido al no volver a confirmar todos los diagnósticos histológicos para la realización de este estudio, aunque estos diagnósticos habían sido analizados previamente para su inclusión en la base de datos. Además, existe la posibilidad de que el estudio histológico de algunos melanomas que se desarrollen sobre un nevo no detecte la presencia del nevo, bien porque este ha sido destruido por el propio melanoma o por no observarlo en los cortes realizados dado que no se agotó el bloque completo en parafina para la valoración histológica. Por otra parte, la robustez de los 
resultados mostrados está avalada por la corrección del valor de la $\mathrm{p}$ para comparaciones múltiples mediante la prueba de Bonferroni.

En nuestro estudio, de la población de pacientes con melanoma cutáneo, el 30,3\% de los pacientes desarrolló un MSN. Este porcentaje es similar al observado en estudios semejantes ${ }^{1,3}$. De todas las variables analizadas, la edad de diagnóstico inferior a 40 años, la localización en el tronco y el melanoma de extensión superficial, fueron las únicas que se encontraron relacionadas con la presentación de un MSN. Además, de éstas, la localización en el tronco fue la que mostró una asociación más fuerte con el desarrollo de un MSN. Algunos autores, han encontrado estos tres mismos factores de riesgo para el desarrollo de un $\mathrm{MSN}^{1,10}$. Incluso Bevona y col. al igual que en nuestro estudio, observaron que la variable más fuertemente asociada al desarrollo de un MSN era la localización en el tronco frente a otras localizaciones ${ }^{3}$. En otro estudio, se obtuvo como variables significativas relacionadas con el desarrollo de un MSN una gran densidad de nevos y al igual que en nuestro estudio la localización en el tronco y el melanoma de extensión superficial, pero no la edad más joven de presentación ${ }^{4}$.

Diversos estudios, han demostrado el riesgo aumentado de desarrollar nuevos melanomas en los pacientes que ya han sido previamente diagnosticados de un melanoma cutáneo ${ }^{5-8}$. Estos segundos melanomas pueden aparecer tanto en el momento del diagnóstico del primer melanoma (sincrónicamente) como durante el seguimiento posterior del paciente (metacrónicamente) y suelen ser más delgados y presentar un menor índice de Clark así como una menor ulceración que en el caso de los primeros melanomas ${ }^{11,12}$. Es por esto por lo que se recomienda mantener un seguimiento durante toda la vida de los pacientes diagnosticados de un melanoma cutáneo.

Tal y como se muestra en el presente estudio, los segundos melanomas al igual que en el caso de los primeros, aparecen con mayor frecuencia de novo, pero aunque en una proporción ligeramente inferior a los primarios $(24,4 \%$ vs 30,3\%) también pueden hacerlo sobre un nevo. Hasta la fecha, no se han estudiado los factores de riesgo asociados al desarrollo de un segundo melanoma sobre una lesión névica previa. Por tanto, este es el primer estudio que evalúa dichos factores.

En nuestro estudio se partía de la hipótesis de que los segundos MSN iban a tener los mismos factores de riesgo que los observados para el desarrollo del primer MSN.

Además, se quiso constatar si el hecho de haber presentado un MSN determinaba de alguna manera la posibilidad de que el segundo melanoma se asociara a un nevo. Por ello, se seleccionaron aquellas variables que habían resultado significativas en el estudio 
univariado como factores de riesgo de presentar un melanoma asociado al primer melanoma. Además, se incluyó como nueva variable el hecho de haber presentado un nevo asociado en el primer melanoma. De todas las variables sólo los pacientes que habían presentado un primer MSN tenían más riesgo de desarrollar un segundo melanoma sobre un nevo previo. Parece probable que la ausencia de la significación de las variables inicialmente asociadas a desarrollar un primer MSN, puede ser debida al tamaño muestral. En cualquier caso, en la serie presentada, el factor más relevante es haber desarrollado el primer melanoma asociado a un nevo, que sí alcanzó significación con este tamaño muestral. Debido al tamaño muestral, no se consideró en el estudio la evaluación por separado del tipo de nevo asociado al melanoma (congénito, adquirido común o adquirido displásico, otros). Una exploración post-hoc de la variable caracterizada de acuerdo con esta clasificación no mostró diferencias en los resultados (datos no mostrados) aunque el reducido número de casos por cada valor de la categoría hace que la valoración de estos resultados sea incierta.

Los datos obtenidos en las dos partes del estudio, apoyan la hipótesis de la existencia de una vía etiopatogénica en el desarrollo del melanoma cutáneo que es el resultado de la inestabilidad de las células pigmentadas. Esta vía, que explicaría el desarrollo del melanoma en pacientes jóvenes, con múltiples nevos y en localizaciones que no están de manera crónica expuestas al sol, como es el tronco, probablemente está implicada en los MSN. 


\section{CONCLUSIONES}

1. Los MSN están asociados a los melanomas localizados en el tronco, en pacientes jóvenes y son del subtipo histológico de extensión superficial.

2. En los pacientes que desarrollan un segundo melanoma, el factor más determinante de que lo hagan asociado a un nevo es que el primer melanoma también se haya desarrollado junto a un nevo.

3. Los pacientes que han presentado un primer melanoma sobre nevo y tienen múltiples nevos serían los que más se beneficiarían de un seguimiento periódico exhaustivo de sus lesiones melánicas, especialmente mediante dermatoscopia digital en centros de referencia.

4. En aquellos casos seleccionados con un número muy escaso de nevos, podría ser interesante evaluar si la extirpación profiláctica de los mismos reduce la incidencia de segundos melanomas, aunque este hecho no se ha demostrado en la población general. 


\section{TABLAS Y GRÁFICOS}

Gráfica 1: Distribución por sexo del primer melanoma

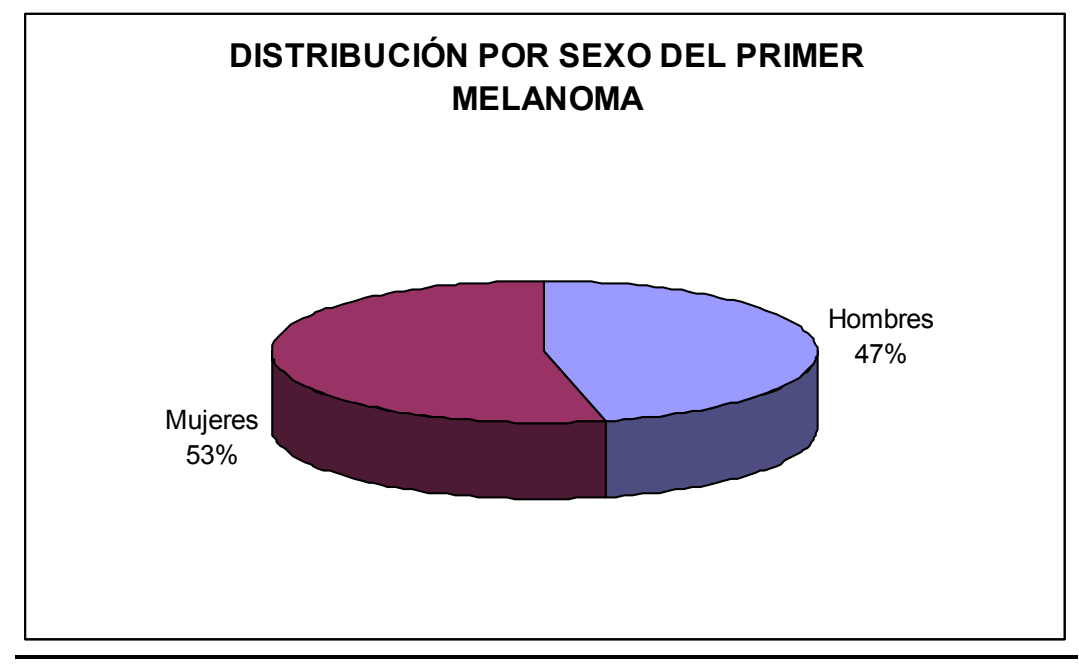

Gráfica 2: Distribución del primer melanoma en función de su asociación o no a un nevo pre-existente.

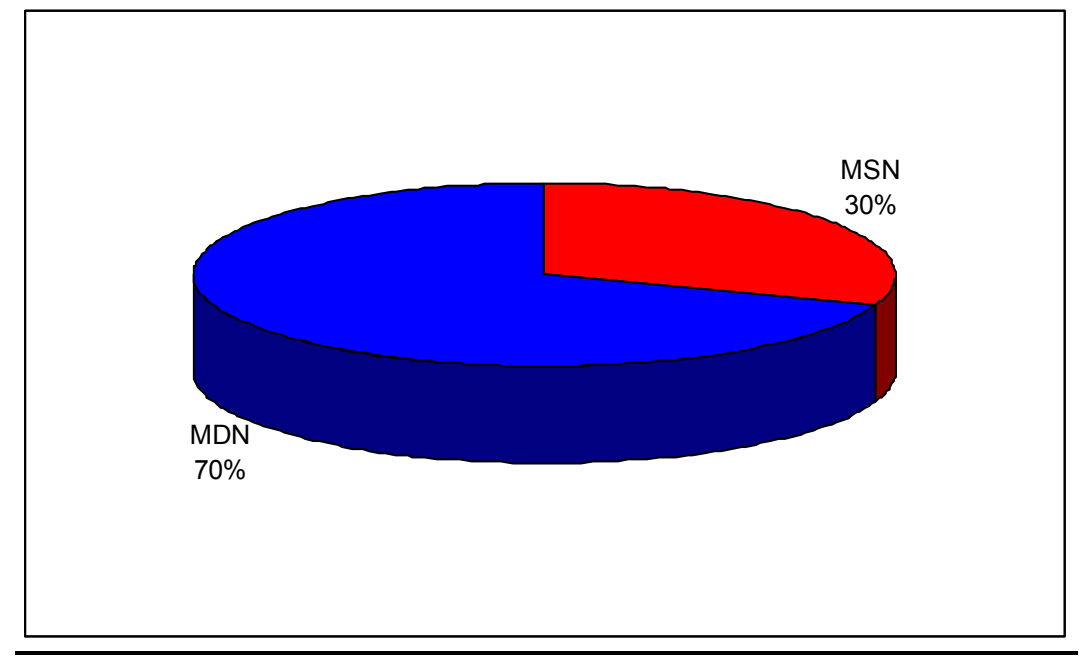


Gráfica 3: Distribución del segundo melanoma en función de su asociación o no a un nevo preexistente.

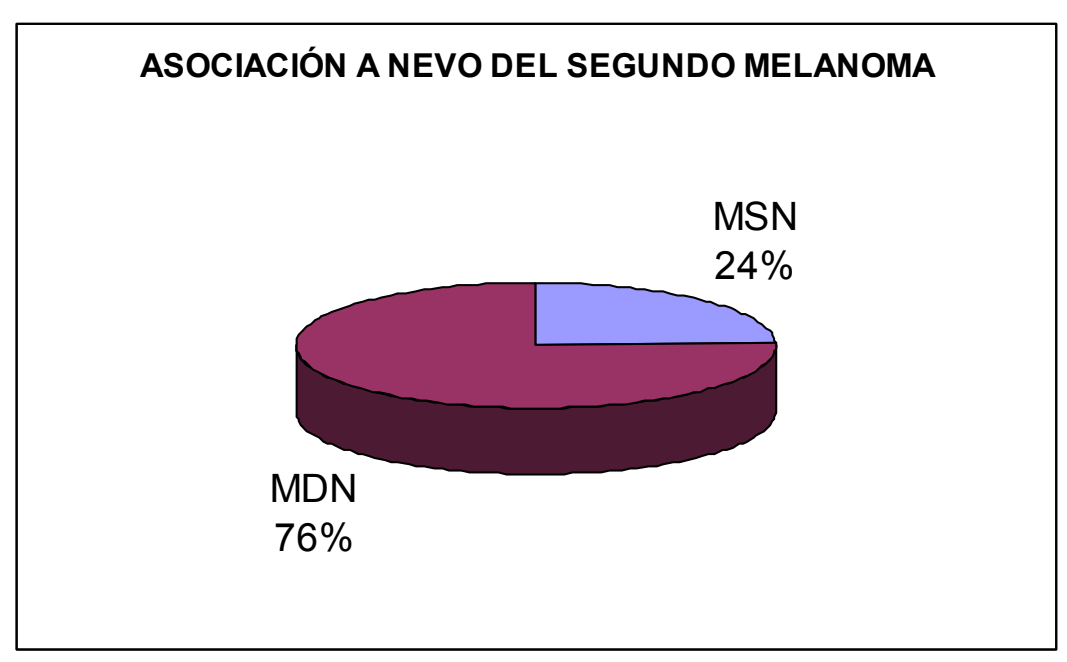

Gráfica 4: Orden de importancia, de acuerdo con el valor de la Ji cuadrado (prueba de Wald) de las variables significativas para el desarrollo de un primer melanoma sobre nevo.

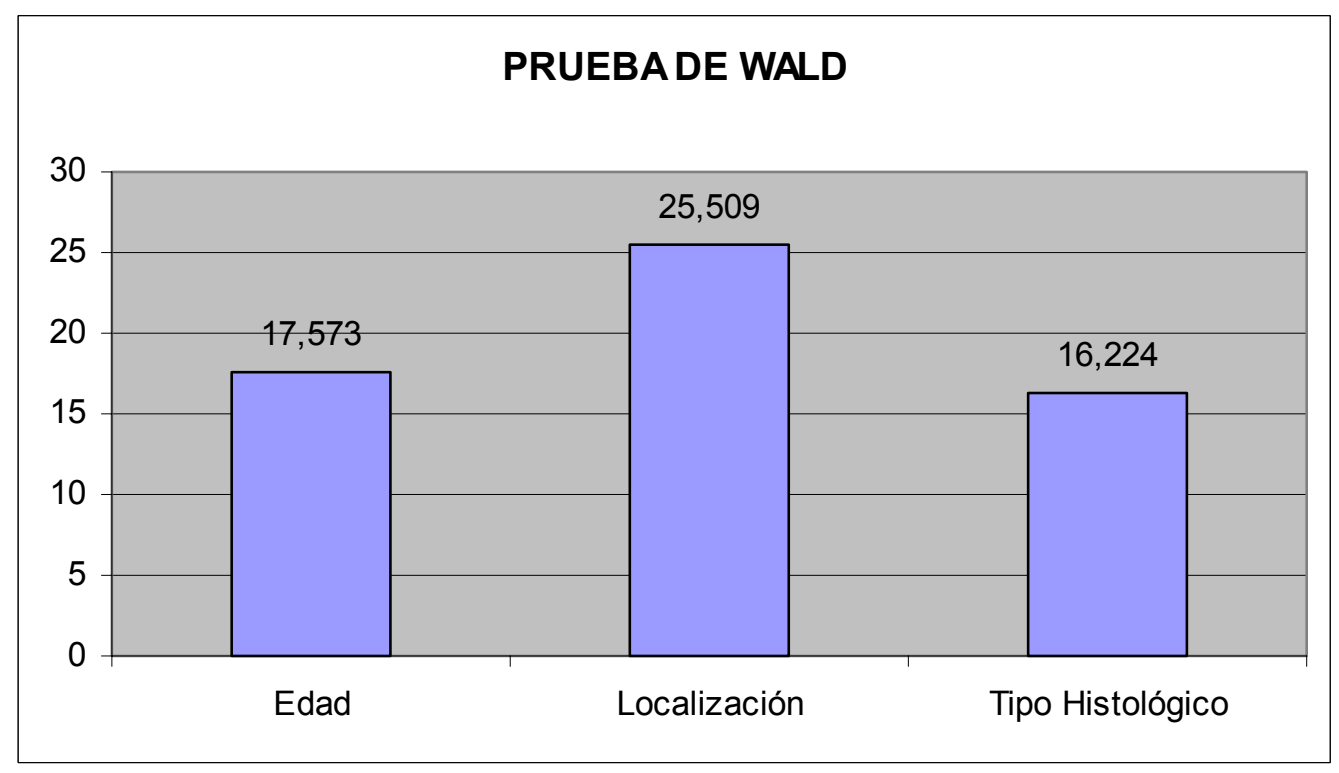




\begin{tabular}{|c|c|c|c|c|c|c|c|}
\hline \multirow{3}{*}{$\begin{array}{l}\text { Característica } \\
\text { Epidemiológicas } \\
\end{array}$} & \multicolumn{7}{|c|}{ Primer melanoma asociado a nevo } \\
\hline & No & $(\%)$ & Sí & $\%$ & PC & OR & IC para EI 95\% Del OR \\
\hline & & & & & & & \\
\hline $\begin{array}{l}\text { Sexo } \\
\text { Hombre } \\
\text { Mujer }\end{array}$ & $\begin{array}{l}319 \\
365\end{array}$ & $\begin{array}{l}(46,6) \\
(53,4)\end{array}$ & $\begin{array}{l}137 \\
160\end{array}$ & $\begin{array}{l}(46,1) \\
(53,9)\end{array}$ & N.S. & 1,02 & $0,78-1,34$ \\
\hline $\begin{array}{l}\text { Edad } \\
<40 \\
40-60 \\
>60\end{array}$ & $\begin{array}{l}133 \\
266 \\
285\end{array}$ & $\begin{array}{l}(19,4) \\
(39,1) \\
(41,7)\end{array}$ & $\begin{array}{r}105 \\
115 \\
77\end{array}$ & $\begin{array}{l}(35,4) \\
(38,7) \\
(25,9)\end{array}$ & 0,0016 & $\begin{array}{c}1 \\
0,55 \\
0,34\end{array}$ & $\begin{array}{c}1 \\
0,39-0,77 \\
0,24-0,49\end{array}$ \\
\hline $\begin{array}{l}\text { Localización } \\
\text { Cabeza/cuello } \\
\text { MMSS } \\
\text { Tronco } \\
\text { MMII } \\
\text { Acral }\end{array}$ & $\begin{array}{r}125 \\
98 \\
231 \\
160 \\
70\end{array}$ & $\begin{array}{l}(18,3) \\
(14,3) \\
(33,8) \\
(23,4) \\
(10,2)\end{array}$ & $\begin{array}{r}32 \\
45 \\
163 \\
45 \\
12\end{array}$ & $\begin{array}{l}(10,8) \\
(15,2) \\
(54,9) \\
(15,2) \\
(4,0)\end{array}$ & 0,0016 & $\begin{array}{c}1 \\
1,79 \\
2,76 \\
1,10 \\
0,67\end{array}$ & $\begin{array}{c}1 \\
1,06-3,03 \\
1,78-4,27 \\
0,66-1,83 \\
0,32-1,38\end{array}$ \\
\hline Fenotípicas & & & & & & & \\
\hline $\begin{array}{l}\text { Fototipo } \\
\text { III-IV } \\
\text { I-II } \\
\end{array}$ & $\begin{array}{l}231 \\
415\end{array}$ & $\begin{array}{l}(35,8) \\
(64,2)\end{array}$ & $\begin{array}{l}119 \\
174\end{array}$ & $\begin{array}{l}(40,6) \\
(59,4)\end{array}$ & N.S. & 1,23 & $\begin{array}{c}1 \\
0,93-1,63\end{array}$ \\
\hline $\begin{array}{l}\text { Ojos } \\
\text { Oscuros } \\
\text { Claros } \\
\end{array}$ & $\begin{array}{l}371 \\
229\end{array}$ & $\begin{array}{l}(61,8) \\
(38,2)\end{array}$ & $\begin{array}{r}179 \\
90\end{array}$ & $\begin{array}{l}(66,5) \\
(33,5)\end{array}$ & N.S. & $\begin{array}{c}1 \\
0,815\end{array}$ & $\begin{array}{c}1 \\
0,60-1,10\end{array}$ \\
\hline $\begin{array}{l}\text { Pelo } \\
\text { Moreno/Castaño } \\
\text { Rubio } \\
\text { Pelirojo }\end{array}$ & $\begin{array}{r}463 \\
111 \\
17 \\
\end{array}$ & $\begin{array}{l}(78,3) \\
(18,8) \\
(2,9) \\
\end{array}$ & $\begin{array}{r}203 \\
48 \\
11 \\
\end{array}$ & $\begin{array}{l}(77,5) \\
(18,3) \\
(4,2) \\
\end{array}$ & N.S. & $\begin{array}{c}1 \\
0,98 \\
1,48 \\
\end{array}$ & $\begin{array}{c}1 \\
0,68-1,44 \\
0,68-3,21 \\
\end{array}$ \\
\hline $\begin{array}{l}\text { Nevos comunes } \\
<20 \\
20-50 \\
51-100 \\
>100\end{array}$ & $\begin{array}{r}362 \\
61 \\
62 \\
29 \\
\end{array}$ & $\begin{array}{l}(70,4) \\
(11,9) \\
(12,1) \\
(5,6)\end{array}$ & $\begin{array}{r}139 \\
39 \\
33 \\
30\end{array}$ & $\begin{array}{l}(57,7) \\
(16,2) \\
(13,7) \\
(12,4)\end{array}$ & N.S. & $\begin{array}{c}1 \\
1,66 \\
1,39 \\
2,70\end{array}$ & $\begin{array}{c}1 \\
1,06-2,60 \\
0,87-2,20 \\
1,56-4,65\end{array}$ \\
\hline $\begin{array}{l}\text { Nevos atípicos } \\
\text { Ninguno } \\
\text { Alguno }\end{array}$ & $\begin{array}{l}495 \\
111\end{array}$ & $\begin{array}{l}(81,7) \\
(18,2)\end{array}$ & $\begin{array}{r}192 \\
83\end{array}$ & $\begin{array}{l}(69,8) \\
(30,2)\end{array}$ & 0,0016 & $\begin{array}{c}1 \\
1,94\end{array}$ & $\begin{array}{c}1 \\
1,40-2,71\end{array}$ \\
\hline $\begin{array}{l}\text { Factores } \\
\text { ambientales }\end{array}$ & & & & & & & \\
\hline $\begin{array}{l}\text { Años exposición } \\
\text { solar laboral } \\
\text { No } \\
</=20 \text { años } \\
>20 \text { años }\end{array}$ & $\begin{array}{r}391 \\
46 \\
76\end{array}$ & $\begin{array}{l}(76,2) \\
(9,0) \\
(14,8)\end{array}$ & $\begin{array}{r}192 \\
20 \\
19\end{array}$ & $\begin{array}{l}(83,1) \\
(8,7) \\
(8,2)\end{array}$ & N.S. & $\begin{array}{c}1 \\
0,89 \\
0,51\end{array}$ & $\begin{array}{c}1 \\
0,51-154 \\
0,30-0,87\end{array}$ \\
\hline $\begin{array}{l}\text { Quemaduras } \\
\text { zona del } \\
\text { melanoma }\end{array}$ & & & & & & & \\
\hline
\end{tabular}




\begin{tabular}{|c|c|c|c|c|c|c|c|}
\hline $\begin{array}{l}\text { No } \\
\text { Leves } \\
\text { Severas } \\
\end{array}$ & $\begin{array}{l}171 \\
205 \\
118 \\
\end{array}$ & $\begin{array}{l}(34,6) \\
(41,5) \\
(23,9) \\
\end{array}$ & $\begin{array}{r}55 \\
103 \\
63 \\
\end{array}$ & \begin{tabular}{|l|}
$(24,9)$ \\
$(46,6)$ \\
$(28,5)$ \\
\end{tabular} & N.S. & $\begin{array}{c}1 \\
1,56 \\
1,66 \\
\end{array}$ & $\begin{array}{c}1 \\
1,06-2,3 \\
1,08-2,55 \\
\end{array}$ \\
\hline $\begin{array}{l}\text { Quemaduras } \\
\text { Intensas } \\
0 \\
1-5 \\
6-10 \\
>10 \\
\end{array}$ & $\begin{array}{r}299 \\
189 \\
63 \\
42\end{array}$ & $\begin{array}{l}(50,4) \\
(31,9) \\
(10,6) \\
(7,1)\end{array}$ & $\begin{array}{r}108 \\
100 \\
40 \\
12\end{array}$ & $\begin{array}{l}(41,5) \\
(38,5) \\
(15,4) \\
(4,6)\end{array}$ & N.S. & $\begin{array}{c}1 \\
1,46 \\
1,76 \\
0,79\end{array}$ & $\begin{array}{c}1 \\
1,06-2,03 \\
1,12-2,77 \\
0,40-1,56\end{array}$ \\
\hline $\begin{array}{l}\text { Antecedentes } \\
\text { familiares de } \\
\text { Melanoma } \\
\text { No } \\
\mathrm{Si} \\
\end{array}$ & $\begin{array}{r}603 \\
37 \\
\end{array}$ & $\begin{array}{l}(94,2) \\
(5,8)\end{array}$ & $\begin{array}{r}274 \\
12 \\
\end{array}$ & $\begin{array}{l}(95,8) \\
(4,2)\end{array}$ & N.S. & $\begin{array}{c}1 \\
0,714 \\
\end{array}$ & $\begin{array}{c}1 \\
0,37-1,4\end{array}$ \\
\hline Histológicas & & & & & & & \\
\hline $\begin{array}{l}\text { Índice de } \\
\text { Breslow } \\
</=1,00 \mathrm{~mm} \\
1,01-4,00 \mathrm{~mm} \\
>4,00 \mathrm{~mm} \\
\end{array}$ & $\begin{array}{r}277 \\
272 \\
89 \\
\end{array}$ & $\begin{array}{l}(43,4) \\
(42,6) \\
(13,9) \\
\end{array}$ & $\begin{array}{r}138 \\
115 \\
15 \\
\end{array}$ & $\begin{array}{l}(51,5) \\
(42,9) \\
(5,6) \\
\end{array}$ & N.S. & $\begin{array}{c}1 \\
0,85 \\
0,34 \\
\end{array}$ & $\begin{array}{c}1 \\
0,63-1,14 \\
0,19-0,60 \\
\end{array}$ \\
\hline $\begin{array}{l}\text { Subtipo } \\
\text { histológico } \\
\text { LMM } \\
\text { MES } \\
\text { MN } \\
\text { MLA } \\
\text { Otros }\end{array}$ & $\begin{array}{r}44 \\
414 \\
154 \\
38 \\
30\end{array}$ & $\begin{array}{l}(6,5) \\
(60,9) \\
(22,6) \\
(5,6) \\
(4,4)\end{array}$ & $\begin{array}{r}7 \\
234 \\
48 \\
3 \\
4\end{array}$ & $\begin{array}{l}(2,4) \\
(79,1) \\
(16,2) \\
(1,0) \\
(1,4)\end{array}$ & 0,0016 & $\begin{array}{c}1 \\
3,55 \\
1,95 \\
0,5 \\
0,84\end{array}$ & $\begin{array}{c}1 \\
1,57-8,01 \\
0,83-4,63 \\
0,12-2,05 \\
0,22-3,11\end{array}$ \\
\hline $\begin{array}{l}\text { OR: Odds ratic } \\
\text { Miembros infe } \\
\text { Bonferroni. N. } \\
\text { de extensión su }\end{array}$ & Inte & $\begin{array}{l}\text { Rvalo d } \\
\text { Valor } \\
\text { ificativ } \\
\text { MN: } m\end{array}$ & $\begin{array}{l}\text { onfi } \\
\text { la p }\end{array}$ & $\begin{array}{l}\text { anza. } \\
\text { tras la } \\
\text { M: Len } \\
\text { la nodu }\end{array}$ & $\begin{array}{l}\text { correce } \\
\text { tigo ma } \\
\text { lar. } \mathrm{Ml}\end{array}$ & $\begin{array}{l}\text { embros } \\
\text { n media } \\
\text { no mela } \\
\text { : melan }\end{array}$ & $\begin{array}{l}\text { res. MMII: } \\
\text { ueba de } \\
\text { IES: melanoma } \\
\text { tiginoso acral. }\end{array}$ \\
\hline
\end{tabular}




\begin{tabular}{|l|c|c|}
\hline $\begin{array}{l}\text { Tabla 2: Modelo multivariado } \\
\text { significativamente asociadas a presentar un primer melanoma sobre nevo }\end{array}$ \\
\hline Variable & OR & IC 95\% del OR \\
\hline Edad & & 1 \\
$<40$ & 1 & $0,41-0,83$ \\
$40-60$ & 0,58 & $0,30-0,66$ \\
$>60$ & 0,45 & 1 \\
\hline Localización & & $0,77-2,35$ \\
Cabeza/cuello & 1 & $1,23-3,19$ \\
MMSS & 1,34 & $0,45-1,37$ \\
Tronco & 1,98 & $0,31-1,87$ \\
MMII & 0,79 & 1 \\
Acral & 0,77 & $0,90-5,10$ \\
\hline Tipo histológico & & $0,54-3,31$ \\
LMM & 1 & $0,13-3,38$ \\
MES & 2,14 & $0,12-1,87$ \\
MN & 1,34 & con \\
MLA & 0,65 & las \\
Otros & 0,48 & \\
\hline
\end{tabular}




\begin{tabular}{|c|c|c|c|c|c|}
\hline & Segundo & melano & & & \\
\hline Característica & No & $(\%)$ & Sí & $\%$ & PC \\
\hline $\begin{array}{l}\text { Edad } \\
<40 \\
40-60 \\
>60\end{array}$ & $\begin{array}{r}8 \\
15 \\
11\end{array}$ & $\begin{array}{l}(23,5) \\
(44,1) \\
(32,4)\end{array}$ & $\begin{array}{l}2 \\
4 \\
5\end{array}$ & $\begin{array}{l}(18,2) \\
(36,4) \\
(45,5)\end{array}$ & N.S. \\
\hline $\begin{array}{l}\text { Nevos atípicos } \\
\text { Ninguno } \\
\text { Alguno }\end{array}$ & $\begin{array}{l}17 \\
16\end{array}$ & $\begin{array}{l}(51,5) \\
(48,5)\end{array}$ & $\begin{array}{l}2 \\
8\end{array}$ & $\begin{array}{l}(20) \\
(80)\end{array}$ & N.S. \\
\hline $\begin{array}{l}\text { Localización } \\
\text { Cabeza/ Cuello } \\
\text { MMSS } \\
\text { Tronco } \\
\text { MMII } \\
\text { acrales }\end{array}$ & $\begin{array}{r}7 \\
3 \\
17 \\
6 \\
1\end{array}$ & $\begin{array}{l}(20,6) \\
(8,8) \\
(50,0) \\
(17,6) \\
(2,9)\end{array}$ & $\begin{array}{l}4 \\
2 \\
4 \\
0 \\
1\end{array}$ & $\begin{array}{l}(36,4) \\
(18,2) \\
(36,4) \\
(0,0) \\
(9,1)\end{array}$ & N.S. \\
\hline $\begin{array}{l}\text { Tipo } \\
\text { histológico } \\
\text { LMM } \\
\text { MES } \\
\text { MN } \\
\text { MLA } \\
\text { Otros }\end{array}$ & $\begin{array}{r}3 \\
23 \\
7 \\
0 \\
1\end{array}$ & $\begin{array}{l}(8,8) \\
(67,6) \\
(20,6) \\
(0,0) \\
(2,9) \\
\end{array}$ & $\begin{array}{l}0 \\
7 \\
2 \\
1 \\
0\end{array}$ & $\begin{array}{l}(0,0) \\
(70) \\
(20) \\
(10) \\
(0,0)\end{array}$ & N.S. \\
\hline $\begin{array}{l}\text { Nevo asociado } \\
\text { al primer } \\
\text { melanoma } \\
\text { No } \\
\text { Sí }\end{array}$ & $\begin{array}{r}31 \\
3\end{array}$ & $\begin{array}{l}(83,8) \\
(37,5)\end{array}$ & $\begin{array}{l}6 \\
5\end{array}$ & $\begin{array}{l}(16,2) \\
(62,5)\end{array}$ & 0,042 \\
\hline $\begin{array}{c}\text { Pc: Valor de } \\
\text { LMM: Len } \\
\text { superficial }\end{array}$ & $\begin{array}{l}\text { a } \mathrm{p} \text { tras la } \\
\text { igo maligi } \\
\mathrm{MN} \text { : mel }\end{array}$ & $\begin{array}{l}\text { correcci } \\
\text { o melan } \\
\text { inoma n }\end{array}$ & 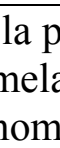 & $\begin{array}{l}\text { ueba de } \\
\text { noma d } \\
\text { a lentigi }\end{array}$ & $\begin{array}{l}\text { rroni. } \\
\text { sión } \\
\text { ral }\end{array}$ \\
\hline
\end{tabular}




\section{BIBLIOGRAFÍA}

1. Whiteman DC, Parsons PG, Green AC. p53 expression and risk factors for cutaneous melanoma: a case-control study. Int J Cancer 1998; 77: 843-8.

2 .Beadling C, Jacobson-Dunlop E, Hodi FS et al. KIT gene mutations and copy number in melanoma subtypes. Clin Cancer Res 2008; 14: 6821-8.

3. Bevona C, Goggins W, Quinn T et al. Cutaneous melanomas associated with nevi. Arch Dermatol 2003; 139: 1620-4; discussion 4.

4. Purdue MP, From L, Armstrong BK et al. Etiologic and other factors predicting nevus-associated cutaneous malignant melanoma. Cancer Epidemiol Biomarkers Prev 2005; 14: 2015-22.

5. DiFronzo LA, Wanek LA, Elashoff R et al. Increased incidence of second primary melanoma in patients with a previous cutaneous melanoma. Ann Surg Oncol 1999; 6: 705-11.

6. Kang S, Barnhill RL, Mihm MC, Jr. et al. Multiple primary cutaneous melanomas. Cancer 1992; 70: 1911-6.

7. Nashan D, Kocer B, Schiller M et al. Significant risk of a second melanoma in patients with a history of melanoma but no further predisposing factors. Dermatology 2003; 206: 76-7.

8. Schmid-Wendtner $\mathrm{MH}$, Baumert J, Wendtner $\mathrm{CM}$ et al. Risk of second primary malignancies in patients with cutaneous melanoma. Br J Dermatol 2001; 145: 981-5.

9. Nagore E, Botella-Estrada R, Requena C et al. Clinical and epidemiologic profile of melanoma patients according to sun exposure of the tumor site. Actas Dermosifiliogr 2009; 100: 205-11. 
10. Carli P, Massi D, Santucci M et al. Cutaneous melanoma histologically associated with a nevus and melanoma de novo have a different profile of risk: results from a casecontrol study. J Am Acad Dermatol 1999; 40: 549-57.

11. Brobeil A, Rapaport D, Wells K et al. Multiple primary melanomas: implications for screening and follow-up programs for melanoma. Ann Surg Oncol 1997; 4: 19-23.

12. DiFronzo LA, Wanek LA, Morton DL. Earlier diagnosis of second primary melanoma confirms the benefits of patient education and routine postoperative followup. Cancer 2001; 91: 1520-4. 